Originalien

Chirurg 2021 · 92:361-368

https://doi.org/10.1007/s00104-020-01257-w

Online publiziert: 5 . August 2020

(c) Der/die Autor(en) 2020

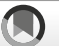

Check for
updates

\section{Hintergrund}

Epilepsie ist eine häufige neurologische Erkrankung, die Patienten jeder Altersklasse betrifft und sich klinisch durch wiederkehrende, meist nichtvorhersagbare epileptische Anfälle unterschiedlicher Semiologie und Ausprägung manifestiert [8, 31]. Im Rahmen epileptischer Anfälle kommt es durch Stürze, Bewusstseinseinschränkungen bzw. unkontrollierbare Muskeltonisierung oder -kloni häufig zu Verletzungen, welche von Hämatomen bis hin zu operativ versorgungspflichtigen Frakturen reichen können $[9,16,33,37,43]$. Retrospektiv berichten ca. $14-18 \%$ der Patienten mit

\section{Author contributions}

L. M. Willems und R. D. Verboket entwickelten die Idee für dieses Projekt und führten die statistische Analyse durch. N. Mühlenfeld führte die Datenerhebung durch. R. D. Verboket, N. Mühlenfeld, J. Sterz, P. Störmann, I. Marzi, F. Rosenow, A. Strzelczyk, und L. M. Willems verfassten den Artikel. Jeder Autor trug wichtige inhaltliche Aspekte bei und stimmte der Publikation des Artikels in der eingereichten Formzu.

René D. Verboket ' Nils Mühlenfeld' · Jasmina Sterz ${ }^{1}$ Philipp Störmann' Ingo Marzi' $\cdot$ Yunus Balcik ${ }^{2,4} \cdot$ Felix Rosenow ${ }^{2,4} \cdot$ Adam Strzelczyk $^{2,3,4}$. Laurent M. Willems $\mathbf{s}^{2,4}$

' Klinik für Unfall-, Hand- und Wiederherstellungschirurgie, Goethe-Universität Frankfurt, Frankfurt am Main, Deutschland

${ }^{2}$ Epilepsiezentrum Frankfurt Rhein-Main und Klinik für Neurologie, Goethe-Universität Frankfurt, Frankfurt am Main, Deutschland

${ }^{3}$ Epilepsiezentrum Hessen und Klinik für Neurologie, Philipps-Universität Marburg, Marburg (Lahn), Deutschland

${ }^{4}$ LOEWE Center for Personalized Translational Epilepsy Research (CePTER), Goethe-Universität Frankfurt, Frankfurt am Main, Deutschland

\title{
Stationäre Versorgungskosten, kostenverursachende Faktoren und potenzielle Vergütungsprobleme bei Verletzungen und Frakturen im Rahmen epileptischer Anfälle
}

Epilepsie über rezente Verletzungen im Rahmen ihrer Anfälle, die Lebenszeitprävalenz anfallsbedingter Verletzungen liegt zwischen 11 und $28 \%$, wobei eine Häufung von Verletzungen bei Patienten mit therapierefraktärer oder schwer $\mathrm{zu}$ behandelnder Epilepsie beschrieben wurde $[4,34,43]$. Insbesondere für Frakturen besteht bei Epilepsiepatienten ein um den Faktor 1,7 bis 6,2 gesteigertes Risiko [39], welches vor allem auf eine reduzierte Knochendichte zurückgeführt wurde $[1,7]$. Häufig bedürfen anfallsbedingte Verletzungen einer unmittelbaren medizinischen Versorgung, z. B. Frakturen, Verbrennungen oder Schnitte, weshalb oftmals eine Vorstellung in Notaufnahmen oder neurologischen bzw. unfallchirurgischen Kliniken zwecks weiterer Versorgung erfolgt [37, 43]. Neben den direkten Traumafolgen gehen Verletzungen im Rahmen epileptischer Anfälle mit einer verminderten Lebensqualität der Betroffenen einher, welche eine weitere relevante Belastung für Patienten und deren Angehörige darstellt [21, 37, $42,43]$.
Die Analyse krankheitsspezifischer Kosten gewinnt in einem zunehmend ökonomisch ausgerichteten Gesundheitssystem mehr und mehr an Relevanz. Aus gesundheitsökonomischer Sicht setzen sich die verletzungsbedingten Kosten im Rahmen epileptische Anfälle aus drei unterschiedlichen Aspekten zusammen: Neben den direkten Versorgungskosten, z.B. ambulante oder stationäre Versorgung bzw. Heil- oder Hilfsmittel, müssen auch indirekte Kosten, z.B. durch Krankschreibung oder Reduktion der Arbeitskraft, sowie intangible Kosten berücksichtigt werden. Letztere summieren die primär nichtmonetären Folgen von Verletzungen, z. B. eine verminderte Lebensqualität, und versuchen diesen einen monetären Gegenwert zuzuschreiben, was bisher kaum adäquat gelingt $[12,28,42]$. Aus Sicht der Behandler sind insbesondere die direkten, also die im Rahmen der ambulanten oder stationären Versorgung anfallenden Krankheitskosten von Relevanz, da diese durch das G-DRG(„German diagnosis related groups")-Vergütungs- 
system kompensiert werden [24]. Über die letzten Jahre wurde das fallbasierte Vergütungssystem immer wieder kritisiert und auf eine mögliche Diskrepanz zwischen erstatteten und tatsächlich angefallenen Kosten hingewiesen [19, 22, 38].

Ziel dieser Studie ist es, die krankheitsspezifischen Kosten der stationären Versorgung anfallsbedingter Verletzungen aufzuarbeiten, wobei neben der Erfassung des Reinerlöses sowie einer Analyse nach kostenverursachenden Faktoren auch eine alternative Kalkulation der Behandlungskosten nach gängigen gesundheitsökonomischen Methoden erfolgte, um potenzielle Vergütungsprobleme des DRG-Systems zu eruieren.

\section{Patienten und Methode}

\section{Studiendesign und Datenakquise}

Diese verwendeten Daten stammen aus einer retrospektiven Studie mit epileptischen Anfällen assoziierten Verletzungen, die 2019 in der Abteilung für Unfall-, Hand- und Wiederherstellungschirurgie am Universitätsklinikum Frankfurt durchgeführt wurde. Mittels einer Abfrage im Krankenhausinformationssystem (KIS) nach den ICD-10-Codes G40.0 bis G40.9 der G-DRG wurden systematisch alle Patienten mit Epilepsie identifiziert, die zwischen Januar 2010 und Januar 2018 stationär in der o.g. Abteilung behandelt wurden. In einem weiteren Schritt wurden alle erfassten Patienten durch zwei unabhängige Untersucher nach akuten Verletzungen im Rahmen epileptischer Anfälle als Vorstellungsursache untersucht. Patienten mit fehlender Assoziation zwischen akuter Verletzung und Anfall wurden ausgeschlossen, ebenso wurde mit unklaren Fällen vorgegangen. Die vorliegende Studie wurde durch die lokale Ethikkommission genehmigt (Frankfurt am Main, Genehmigung 52/18) und folgt den STROBE-Richtlinien für Beobachtungsstudien (Strengthening the Reporting of Observational Studies in Epidemiology) sowie den RECORD-Richtlinien für Observationsstudien (Reporting of Studies Conducted Using Observational Routinely-collected Data; $[5,13])$. Ein ähnliches Studiendesign wurde bereits für die gleiche Fragestellung bei Patienten mit Morbus Parkinson verwendet $[17,38]$.

\section{Kostenkalkulation}

Der DRG-Erlös jedes Falls wurde erfasst und als tatsächlich vergütete Kosten gewertet. Entsprechend den Empfehlungen zur Kalkulation stationärer Behandlungskosten wurde für die alterative Berechnung auf abteilungs- und klinikspezifische Tagessätze zurückgegriffen [3, 15]. Im Jahr 2011 wurden Pauschalen von $593,40 €$ für Normalstationen und $1337,72 €$ für Intensivstationen empfohlen [3], welche Pflege, Medikation und Diagnostik beinhalten. Analog zu anderen gesundheitsökonomischen Analysen wurden die Pauschalen für die Jahre 2012 bis 2018 um die Inflation im medizinischen Sektor korrigiert [11, 32, 38, 42]. Die zusätzlich anfallenden Kosten für die operative Versorgung von Frakturen wurden nach Waeschle et al. berechnet, welche im Jahr 2016 die Kosten pro Operationsminute systematisch und DRGkonform analysiert haben. Der vorliegenden Berechnung liegen die über alle Fachabteilungen gemittelten Kosten von $16,63 €$ pro Minute zugrunde [41], welche ebenfalls um die Inflationsraten im medizinischen Bereich angepasst wurden. Aufgrund der Berechnung krankheitsspezifischer Versorgungskosten nach pauschalisiertem Ansatz wurden, verglichen mit einem Micro-costing-Ansatz, keine räumlichen, apparativen oder personellen Anschaffungs- bzw. Vorhaltekosten berücksichtigt.

\section{Datenverarbeitung und Statistik}

Die Aufarbeitung und statistische Auswertung der Daten erfolgte unter Verwendung von IBM SPSS Statistics 25 (SPSS Inc., Armonk, NY, USA). Die statistische Aufarbeitung der DRG-Erlöse erfolgte aufgrund einer rechtsschiefen Verteilung der anfallenden Kosten unter Verwendung der Bootstrap-Methode [35] unter Angabe von Mittelwert, Standardabweichung, Minimum, Maximum, Median sowie des 95\%-Konfidenzintervalls. Die univariate Analyse von mit höheren Kosten assoziierten Faktoren erfolgte unter einheitlicher Verwendung des Eta-Koeffizienten $(\eta)$ bei Verwendung je eines metrisch- (Kosten) und eines nominalskalierten Parameters. Aus der Berechnung resultiert ein Koeffizient zwischen 0 (keine Korrelation) und 1 (sehr starke Korrelation). Hierbei zeigt ein $\eta<0,01$ keine Korrelation an, ein $\eta$ zwischen 0,01 und 0,04 eine geringe, ein $\eta$ zwischen 0,04 und 0,16 eine mittelstarke und ein $\eta$ von $>0,16$ eine starke Korrelation [26]. Zudem erfolgt die Angabe von Somers' d zur weiteren Beschreibung der Korrelation sowie zur Analyse einer signifikanten Korrelation [18, 26, 30]. Die univariate Analyse hinsichtlich potenzieller Vergütungsprobleme erfolgte in gleicher Weise basierend auf der Differenz zwischen kalkulierten Kosten und tatsächlichem Erlös. Alle Ergebnisse wurden nach der Benjamini-HochbergKorrektur für multiples Testen korrigiert [2].

\section{Ergebnisse}

\section{Studienpopulation, stationärer Aufenthalt, Verletzungen und Versorgung}

Von insgesamt 264 Patienten mit Epilepsie als Nebendiagnose in der initialen Erhebung konnten bei 62 Patienten $(23,48 \%)$ eine Verletzung im Rahmen eines epileptischen Anfalls als sichere Ursache identifiziert werden. Alle identifizierten Patienten gingen in die finale Auswertung ein. Das Durchschnittsalter der Kohorte betrug 58,1 Jahre $( \pm 17,8$ Jahre, Median 57 Jahre, Spannweite 21-86 Jahre), 59,7 $\%$ waren weiblich $(n=37)$ und $40,3 \%$ männlich ( $n=25$; - Tab. 1$)$. Betrachtet man das Verletzungsmuster so zeigte sich mit 40,3\% $(n=25)$ der Fälle ein Schädel-Hirn-Trauma als häufigster Grund für die stationäre Aufnahme und Überwachung. Nachrangig folgend waren Frakturen der oberen Extremität $32,3 \%(n=20)$ und Weichteilverletzungen $30,6 \%(n=19)$ Gründe für stationäre Aufnahmen (• Tab.2). Die mittlere Aufenthaltsdauer im Krankenhaus betrug bei den ausgewerteten Patienten 11,4 Tage $( \pm 12,4$ Tage, Median 7 Tage, Spannweite 1-64 Tage). Bei den Patienten mit Frak- 
Chirurg 2021 · 92:361-368 https://doi.org/10.1007/s00104-020-01257-w

(c) Der/die Autor(en) 2020

R. D. Verboket · N. Mühlenfeld · J. Sterz · P. Störmann · I. Marzi · Y. Balcik · F. Rosenow · A. Strzelczyk · L. M. Willems

\section{Stationäre Versorgungskosten, kostenverursachende Faktoren und potenzielle Vergütungsprobleme bei Verletzungen und Frakturen im Rahmen epileptischer Anfälle}

\section{Zusammenfassung}

Hintergrund. Die Analyse krankheitsspezifischer Kosten gewinnt in einem zunehmend ökonomisch ausgerichteten Gesundheitssystem an Relevanz, wobei vor allem chronische Erkrankungen aufgrund der langen Krankheitsdauer sowie häufiger Hospitalisierung und Arztbesuche von besonderem Interesse sind. Epilepsien stellen eine häufige neurologische Erkrankung dar, welche mit paroxysmal auftretenden epileptischen Anfällen und häufig hiermit assoziierten Verletzungen einhergeht und alle Altersgruppen betrifft.

Ziel. Ziel der Arbeit ist die Aufarbeitung der stationären Behandlungskosten anfallsbedingter Verletzungen sowie die Analyse hinsichtlich relevanter kostenverursachender Faktoren. Mittels alternativer Kalkulation der Versorgungskosten soll zusätzlich der Frage nach potenziellen Vergütungsproblemen im aktuellen DRG-System (, diagnosis related groups") nachgegangen werden.
Methoden. Grundlage dieser monozentrischen, retrospektiven Analyse ist der tatsächliche Erlös der stationären Behandlung von 62 Patienten, die zwischen 01/2010 und 01/2018 im Universitätsklinikum Frankfurt aufgrund von Verletzungen im Rahmen epileptischer Anfälle erfolgte. Die Analyse potenzieller kostenverursachender Faktoren bezog sich auf relevante soziodemographische und klinische Aspekte, die alternative Kalkulation der Versorgungskosten wurde mit gängigen gesundheitsökonomischen Methoden durchgeführt.

Ergebnisse. Der mittlere DRG-Erlös betrug $7408 €( \pm 8993 €$, Median $5086 €$, Spanne 563-44.519€), die mittleren kalkulierten Kosten $9423 €( \pm 11.113 €, 5626 €$, Spanne 587-49.830€). Als signifikant kostenverursachender Faktor konnte eine Liegedauer $\geq 7$ Tage $(p=0,014)$ identifiziert werden. Aufgrund des signifikanten Unterschieds $(p<0,001)$ zwischen Erlös und kalkulierten
Kosten erfolgte eine Analyse nach Faktoren für potenzielle Vergütungsprobleme, welche für eine Aufenthaltsdauer von $\geq 7$ Tagen $(p=0,014)$ sowie für eine Behandlung auf Intensivstation $(p=0,019)$ signifikant verblieb. Schlussfolgerung. Die stationären Versorgungskosten von Patienten mit Frakturen aufgrund epileptischer Anfälle sind hoch und daher gesundheitsökonomisch relevant. Generell scheint die auf Fallpauschalen basierende Vergütung nach G-DRG die tatsächlichen Kosten zu decken, bei Patienten mit einer langen Liegedauer oder einen Aufenthalt auf Intensivstation können jedoch Vergütungsprobleme bestehen.

Schlüsselwörter Gesundheitsökonomie · Krankheitskosten · DRG · Epilepsie · Lebensqualität

\section{Inpatient treatment costs, cost-driving factors and potential reimbursement problems due to epileptic seizure-related injuries and fractures}

\section{Abstract}

Background. The systematic analysis of disease-specific costs is becoming increasingly more relevant in an economically oriented healthcare system. Chronic diseases are of particular interest due to the long duration as well as frequent hospitalization and physician visits. Epilepsy is a frequent neurological disorder affecting all age groups with the clinical hallmark of paroxysmal epileptic seizures, which are often associated with injuries.

Objective. The aim of this work was to process the inpatient treatment costs due to seizure-related injuries and fractures. Moreover, relevant cost-causing factors were addressed. Using an alternative calculation of the costs of care, the question of potential reimbursement problems in the current German diagnosis-related groups (G-DRG) system was additionally assessed.
Methods. For this monocentric retrospective analysis the actual proceeds of 62 inpatients who were treated at the University Hospital Frankfurt between January 2010 and January 2018 for injuries and fractures due to epileptic seizures were used. The analysis of potential cost-causing factors was carried out with respect to relevant sociodemographic and clinical aspects. The alternative calculation of the costs of treatment was carried out using established health economic methods.

Results. The average DRG revenue was $7408 €$ ( $\pm 8993 €$, median 5086€, range 563-44,519€), the average calculated costs were $9423 €$ ( $\pm 11,113 €, 5626 €$, range $587-49,830 €)$. A length of stay $\geq 7$ days $(p=0.014)$ was identified as a significant cost-driving factor. Due to the significant difference $(p<0.001)$ between revenue and calculated costs, an analysis was made according to factors for potential reimbursement problems, which remained significant for a length of stay of $\geq 7$ days $(p=0.014)$ and for treatment in the intensive care unit $(p=0.019)$.

Conclusion. The inpatient treatment costs for patients with injuries and fractures due to epileptic seizures are high and therefore relevant from a health economic perspective. In general, reimbursement according to the G-DRG appears to cover the actual costs, but there may be reimbursement problems for patients with a long period of hospitalization or a stay in an intensive care ward.

Keywords

Health economics - Treatment costs · Diagnosis related groups - Epilepsy - Quality of life 


\begin{tabular}{|c|c|}
\hline Geschlecht & $\%(n)$ \\
\hline Weiblich & $59,7(37)$ \\
\hline Männlich & $40,3(25)$ \\
\hline Alter & (Jahre) \\
\hline Mittelwert $\pm S A$ & $58,1 \pm 17,8$ \\
\hline Median & 57,0 \\
\hline Spannweite & $21-86$ \\
\hline \multicolumn{2}{|c|}{ SA Standardabweichung } \\
\hline
\end{tabular}

turen konnte in 54,8\% der Fälle $(n=34)$ konservativ vorgegangen werden, $45,2 \%$ $(n=28)$ mussten operativ versorgt werden. In 29,0\% der Fälle ( $n=18)$ musste eine Behandlung auf einer Intensivstation erfolgen. Die genauen Verweildauern für Patienten mit und ohne Frakturen auf Normal- und Intensivstationen sind in - Tab. 2 dargestellt.

\section{Tatsächlicher Erlös und kalkulierte Kosten}

Der tatsächliche mittlere DRG-Erlös der analysierten Fälle betrug $7408 €( \pm 8993 €$, Median $5008 €)$. In der Kalkulation der krankheitsspezifischen Behandlungskosten zeigte sich im Mittel ein Betrag von $9423 €( \pm 11.113 €$, Median 5626€), wobei der Größte Anteil der angefallenen Kosten der stationäre Aufenthalt aus machte $(8916 €, \pm 11.064 €$, Median $4851 €$, • Tab. 3).

\section{Univariate Analyse kostenverursa- chender Faktoren}

In der Analyse kostenverursachender Faktoren zeigte sich nach Korrektur für multiples Testen eine starke $(\eta>0,16)$ signifikante Korrelation erhöhter direkter Kosten mit einer Aufenthaltsdauer von $\geq 7$ Tage $(p=0,014)$. Eine nicht signifikante, aber starke $(\eta>0,16)$ Korrelation erhöhter direkter Kosten fand sich für männliches Geschlecht, ein Alter $\geq 65$ Jahre sowie für eine intensivmedizinische Behandlung, eine nicht signifikante geringe oder mittelstarke $(\eta>0,04)$ Korrelation für eine operative Versorgung, eine Fraktur generell sowie der oberen Extremität oder des Körperstamms oder Schädels, eine anti-

Tab. 2 Anfallsbedingte Verletzungen sowie Charakteristika von Aufenthalt und Versorgung

\begin{tabular}{|c|c|c|c|c|}
\hline Verletzungsmuster $^{\mathrm{a}}$ & \multicolumn{4}{|c|}{$\%(n)$} \\
\hline Weichteilverletzungen & \multicolumn{4}{|c|}{$30,6(19)$} \\
\hline Fraktur obere Extremität & \multicolumn{4}{|c|}{$32,3(20)$} \\
\hline Fraktur untere Extremität & \multicolumn{4}{|c|}{$8,1(5)$} \\
\hline Fraktur Körperstamm & \multicolumn{4}{|c|}{$24,2(15)$} \\
\hline Schädel-Hirn-Trauma & \multicolumn{4}{|c|}{$40,3(25)$} \\
\hline Fraktur Schädel & \multicolumn{4}{|c|}{$9,7(6)$} \\
\hline Krankenhausaufenthalt (Tage) & MW & $\pm \mathrm{SA}$ & MED & SW \\
\hline \multicolumn{5}{|l|}{ Alle Patienten } \\
\hline Gesamtdauer & 11,4 & 12,4 & 7,0 & $1,0-64,0$ \\
\hline Normalstation & 10,8 & 12,2 & 7,0 & $1,0-64,0$ \\
\hline Intensivstation & 1,7 & 4,2 & 0,0 & $0,0-24,0$ \\
\hline \multicolumn{5}{|l|}{ Patienten mit Fraktur } \\
\hline Gesamtdauer & 12,4 & 13,1 & 7,0 & $2,0-64,0$ \\
\hline Normalstation & 12,2 & 13,1 & 7,0 & $2,0-64,0$ \\
\hline Intensivstation & 1,4 & 1,4 & 0,0 & $0,0-18,0$ \\
\hline \multicolumn{5}{|l|}{ Patienten ohne Fraktur } \\
\hline Gesamtdauer & 9,2 & 10,7 & 2,0 & $1,0-31,0$ \\
\hline Normalstation & 7,6 & 9,6 & 2,0 & $1,0-31,0$ \\
\hline Intensivstation & 2,3 & 5,7 & 0,0 & $0,0-24,0$ \\
\hline Versorgung & \multicolumn{4}{|c|}{$\%(n)$} \\
\hline Aufenthalt Intensivstation & \multicolumn{4}{|c|}{$29,0(18)$} \\
\hline Frakturversorgung operativ & \multicolumn{4}{|c|}{$45,2(28)$} \\
\hline Frakturversorgung konservativ & \multicolumn{4}{|c|}{$54,8(34)$} \\
\hline
\end{tabular}

konvulsive Mehrfachtherapie, das Vorliegen einer aktiven Epilepsie sowie eine generalisiert tonisch-klonische Anfallssemiologie. Keine Korrelation $(\eta \leq 0,04)$ in Bezug auf die direkten Behandlungskosten fand sich für das Vorliegen eine Schädel-Hirn-Traumas oder das Vorhandensein einer Fraktur der unteren Extremität (s. • Tab. 4).

\section{Univariate Analyse potenzieller Faktoren für Vergütungsprobleme}

Die univariate Analyse nach potenziellen Faktoren für Vergütungsprobleme zeigte eine signifikante, starke $(\eta>0,16)$ Korrelation der Diskrepanzen zwischen kalkulierten Kosten und tatsächlichem Erlös in Bezug auf eine Aufenthaltsdauer von $>7$ Tagen $(p=0,014)$ sowie für einen Aufenthalt auf Intensivstation $(p=0,019)$. Eine starke $(\eta>0,16)$, nicht signifikante Korrelation fand sich für ein Alter $\geq 65$ Jahre sowie für eine Fraktur des Schädels. Eine nicht signifi- kante geringe oder mittelstarke $(\eta>0,04)$ Korrelation zeigte sich für männliches Geschlecht, eine operative Versorgung, das Vorhandensein einer Fraktur generell sowie für eine Fraktur der oberen und unteren Extremität und des Körperstamms sowie für eine antikonvulsive Mehrfachtherapie, eine Anfallsfrequenz von $\geq 1$ in den letzten 12 Monaten und das Vorhandensein einer generalisiert tonisch-klonischen Anfallssemiologie (s. - Tab. 4).

\section{Diskussion}

Die Analyse krankheitsspezifischer Kosten gewinnt in einem mehr und mehr ökonomisch ausgerichteten Gesundheitssystem zunehmend an Relevanz. Diese Tendenz einer Ökonomisierung der Medizin wurde über die letzten Jahre kontrovers diskutiert und kritisiert $[14,27,29,40]$. Unabhängig davon, ob man diese Tendenzen als positiv oder negativ betrachtet, resultiert aus 
Tab. 3 Tatsächlicher Erlös und kalkulierte Kosten

\begin{tabular}{|c|c|c|c|c|c|c|}
\hline \multirow[t]{2}{*}{ Einzelposten } & \multicolumn{6}{|c|}{ Kostenberechnung } \\
\hline & MW (€) & $\pm \mathrm{SA}(\epsilon)$ & MED (€) & $\operatorname{MIN}(\boldsymbol{\epsilon})$ & $\operatorname{MAX}(\epsilon)$ & $95 \%-K I(€)$ \\
\hline $\begin{array}{l}\text { Kalkulierte Gesamtkosten } \\
\text { Kosten }\end{array}$ & 9423 & 11.113 & 5626 & 587 & 49.830 & 6998-11.749 \\
\hline $\begin{array}{l}\text { Kalkulierte stationäre Versor- } \\
\text { gung }\end{array}$ & 8916 & 11.064 & 4851 & 4983 & 49.830 & 6517-11.313 \\
\hline Normalstation & 6531 & 7380 & 4143 & 587 & 38.618 & $4902-8582$ \\
\hline Intensivstation & 2384 & 5971 & 0 & 0 & 33.806 & $1208-3839$ \\
\hline $\begin{array}{l}\text { Kalkulierte operative Versor- } \\
\text { gung }\end{array}$ & 507 & 635 & 0 & 0 & 1856 & $349-656$ \\
\hline DRG Erlös & 7408 & 8993 & 5008 & 563 & 44.519 & 5353-9957 \\
\hline
\end{tabular}

der zunehmenden Ökonomisierung der medizinischen Versorgung insbesondere für die erstversorgenden Instanzen, z. B. Notaufnahmen, Ambulanzen oder Kliniken, ein intrinsisches und existenzielles Interesse, die tatsächlich angefallenen Kosten durch die fallbezogene Vergütung nach G-DRG-System zu decken. Immer wieder wurde beschrieben, dass in Bezug auf spezielle Krankheitsbilder die tatsächlichen Kosten durch den DRG-Erlös nicht zwangsläufig zu decken sind [22, 38], was die Kalkulation der tatsächlich anfallenden Kosten zu einer zentralen gesundheitsökonomischen Aufgabe macht [38, 42].

Gesundheitsökonomisch besonders relevant ist hierbei die Erfassung der Kosten häufiger chronischer Erkrankungen, da diese im Hinblick auf die Gesamtausgaben des Gesundheitssystems einen beträchtlichen Teil ausmachen. Die krankheitsspezifischen Kosten für neurologische Erkrankungen beliefen sich im Jahr 2019 laut Statistischem Bundesamt auf 17,2 Mrd. $€$, wovon wiederum 1,6 Mrd. $€(9,3 \%)$ auf Patienten mit Epilepsie bzw. epileptischen Anfällen zurückzuführen sind [6]. Hierbei teilen sich die krankheitsspezifischen Kosten, welche zuletzt mit $14.500 €$ pro Jahr für Patienten mit aktiver Epilepsie und $2500 €$ für anfallsfreie Patienten pro Jahr kalkuliert wurden, in etwa zu gleichen Teilen auf direkte und indirekte Kosten auf, wobei ein Trend über die letzten Jahre hinsichtlich eines zunehmenden Anteils indirekter Kosten zu beobachten ist $[20,42,44]$.
Ein häufiger notfälliger Vorstellungsgrund von Patienten mit Epilepsie oder einem epileptischen Anfall in unfallchirsind in der Regel anfallsassoziierte Unfälle und Verletzungen (,epilepsy related injuries and accidents" [ERIA]), für welche eine jährliche Prävalenz von 14-18 \% und eine Lebenszeitprävalenz zwischen 11 und $28 \%$ beschrieben wurde [4, 43]. Aufgrund einer hohen Dunkelziffer fälschlich nicht kausal auf epileptische Anfälle zurückgeführte Verletzungen ist von einer deutlich höheren Gesamtprävalenz auszugehen [10], was die ökonomische Relevanz anfallsbedingter Verletzungen unterstreichet.

Der tatsächliche mittlere DRG-Erlös der untersuchten Kohorte belief sich auf $7408 €$, was bei einer mittleren Verweildauer von 12 Tagen $617 €$ Erlös pro Tag bedeutet. Hierbei konnte eine signifikante Korrelation der Verweildauer mit dem Erlös nachgewiesen werden. Die alternative Kalkulation der angefallenen krankheitsspezifischen Kosten mittels Tagessätzen und Verweildauer auf Intensiv- und Normalstation sowie minutengenauer Vergütung der Operationsdauer, wenn zutreffend, ergab einen Betrag von $9423 €$, entsprechend $785 €$ pro Tag, entsprechend einer Differenz von täglich $168 €$, entsprechend einem signifikanten Unterschied zum tatsächlichen Erlös. Die Analyse hinsichtlich potenzieller Faktoren, die diese Vergütungsproblem darstellen könnten, zeigte eine signifikante Diskrepanz insbesondere bei einer stationären Verurgischen oder orthopädischen Kliniken Abweichung bedingen und somit ein weildauer von $\geq 7$ Tagen sowie einem Aufenthalt auf Intensivstation. Bezüglich einer längeren Aufenthaltsdauer als ein potenzieller kostenverursachender Faktor passt das vorliegende Ergebnis mit einer rezenten Untersuchung der stationären Versorgungskosten bei Patienten mit Frakturen im Rahmen von Stürzen bei idiopathischem ParkinsonSyndrom sowie weiteren gesundheitsökonomischen Arbeiten überein [25, 36, 38, 45]. Dies mag prinzipiell auf einen Zusammenhang von Vergütungsproblemen beim Überschreiten der Obergrenze der mittleren Verweildauer hinweisen. Das Prinzip der mittleren Verweildauer mit einer Obergrenze wurde bereits kurz nach Einführung der DRG-Pauschalen auf dem Gebiet der Unfallchirurgie und Orthopädie deutlich kritisiert und Nachbesserungen bei der Vergütung gefordert [23]. Obwohl diese zwischenzeitlich erfolgt sind, erscheint insbesondere bei langer Liegedauer bei Patienten mit Verletzungen durch epileptische Anfälle jedoch weiterhin ein Vergütungsproblem zu bestehen. Auch die Diskrepanz bezüglich der Vergütung von Patienten mit intensivmedizinischer Behandlung wurde bereits generell sowie bei anderen Krankheitsbildern beschrieben [22] und verweist auf eine mögliche Unterrepräsentation dieser Fälle in der aktuellen pauschalisierten Vergütung nach G-DRG. Im Hinblick auf beide Aspekte sind weitere versorgungsmedizinische Analysen notwendig, um ggf. eine weitere Anpassung der Vergütung begründen zu können.

Trotz Beachtung der STROBE- und RECORD-Richtlinien für Beobachtungsstudien bzw. Observationsstudien unterliegt diese Studie aufgrund ihres Designs potenziellen Limitationen [5, 13]. Neben der begrenzten Anzahl an analysierten Patienten, welche jedoch in Anbetracht des orientierenden Charakters der Studie ausreichend erscheint, schränkt auch das retrospektive, monozentrische Design der Studie die Interpretation der Daten ein. Die Anpassung der Ausgangskosten für die alternative Kalkulation der Versorgungskosten durch Anpassung nach Inflation im medizinischen Sektor folgt den aktuell gebräuchlichen Methoden, verzerrt die 
Tab. 4 Univariate Analyse kostenverursachender Faktoren sowie von Faktoren für potenzielle Vergütungsprobleme

\begin{tabular}{|c|c|c|c|c|c|c|c|c|c|c|c|c|}
\hline \multirow[t]{2}{*}{ Faktor } & \multicolumn{2}{|c|}{ Kohorte } & \multicolumn{5}{|c|}{ Kostenverursachende Faktoren } & \multicolumn{5}{|c|}{ Potenzielle Faktoren für Vergütungsprobleme } \\
\hline & & $\%(n)$ & MW (€) & $\pm \mathrm{SA}(\epsilon)$ & $\eta^{a}$ & $d^{a}$ & $p$-Wert & MW (€) & $\pm S A(€)$ & $\eta^{a}$ & $d^{a}$ & $p$-Wert \\
\hline \multicolumn{13}{|l|}{ Demographie } \\
\hline \multirow[t]{2}{*}{ Geschlecht } & w & $35,5(22)$ & 6,818 & 7,924 & \multirow[t]{2}{*}{0,175} & \multirow[t]{2}{*}{0,122} & \multirow[t]{2}{*}{0,091} & 3,391 & 5,066 & \multirow[t]{2}{*}{0,074} & \multirow[t]{2}{*}{0,093} & \multirow[t]{2}{*}{0,434} \\
\hline & $\mathrm{m}$ & $64,5(40)$ & 10,856 & 12,400 & & & & 4,254 & 5,979 & & & \\
\hline \multirow[t]{2}{*}{ Alter } & $<65$ & $58,1(36)$ & 7,339 & 8,549 & \multirow[t]{2}{*}{0,222} & \multirow[t]{2}{*}{0,154} & \multirow[t]{2}{*}{0,115} & 2,884 & 566 & \multirow[t]{2}{*}{0,224} & \multirow[t]{2}{*}{0,139} & \multirow[t]{2}{*}{0,220} \\
\hline & $>65$ & $41,9(26)$ & 12,307 & 13,588 & & & & 5,420 & 6,586 & & & \\
\hline \multicolumn{13}{|l|}{ Versorgung } \\
\hline \multirow{2}{*}{$\begin{array}{l}\text { Operative } \\
\text { Versorgung }\end{array}$} & Ja & $45,2(28)$ & 9,995 & 9,657 & \multirow[t]{2}{*}{0,047} & \multirow[t]{2}{*}{$-0,159$} & \multirow[t]{2}{*}{0,117} & 3,424 & 3,940 & \multirow[t]{2}{*}{0,085} & \multirow[t]{2}{*}{$-0,121$} & 0,280 \\
\hline & Nein & $54,8(34)$ & 8,951 & 12,917 & & & & 4,379 & 6,766 & & & \\
\hline Intensivs- & $\mathrm{Ja}$ & $29,0(18)$ & 19,130 & 14,091 & 0,563 & $-0,169$ & 0,117 & 7,826 & 7,638 & 0,787 & 0,244 & 0,019 \\
\hline tation & Nein & $71,0(44)$ & 5,452 & 6,435 & & & & 2,361 & 3,642 & & & \\
\hline Aufenthalts- & $<7 d$ & $45,2(28)$ & 2,519 & 2,150 & 0,479 & 0,427 & 0,014 & 930 & 909 & 0,488 & 0,377 & 0,014 \\
\hline & $\geq 7 d$ & $54,8(34)$ & 15,108 & 12,295 & & & & 6,425 & 6,650 & & & \\
\hline Verletzung & & & & & & & & & & & & \\
\hline Fraktur & Ja & $69,4(43)$ & 10,098 & 10,416 & 0,092 & $-0,169$ & 0,117 & 4,148 & 5,906 & 0,054 & $-0,099$ & 0,405 \\
\hline & Nein & $30,6(19)$ & 7,893 & 12,749 & & & & 3,494 & 5,127 & & & \\
\hline Fraktur obere & Ja & $32,3(20)$ & 7,058 & 6,221 & 0,151 & $-0,004$ & 0,974 & 3,035 & 3,665 & 0,114 & $-0,069$ & 0,465 \\
\hline Extremität & Nein & $66,1(42)$ & 10,639 & 12,876 & & & & 4,404 & 6,448 & & & \\
\hline Fraktur unte- & Ja & $8,1(5)$ & 10,920 & 10,925 & 0,040 & $-0,200$ & 0,807 & 6,280 & 8,137 & 0,123 & $-0,303$ & 0,640 \\
\hline re extremitat & Nein & $91,9(57)$ & 9,291 & 11,226 & & & & 3,743 & 5,427 & & & \\
\hline Fraktur & Ja & $24,2(15)$ & 11,745 & 10,511 & 0,119 & $-0,102$ & 0,280 & 3,294 & 4,512 & 0,066 & 0,023 & 0,852 \\
\hline Korperstamm & Nein & $75,8(47)$ & 8,682 & 11,319 & & & & 4,156 & 5,989 & & & \\
\hline Fraktur & Ja & $9,7(6)$ & 14,108 & 17,949 & 0,139 & $-0,043$ & 0,465 & 6,889 & 10,755 & 0,172 & $-0,021$ & 0,807 \\
\hline Schadel & Nein & $90,3(56)$ & 9,821 & 10,260 & & & & 3,633 & 4,876 & & & \\
\hline Schädel-Hirn- & Ja & $40,3(25)$ & 9,706 & 14,321 & 0,021 & 0,120 & 0,285 & 4,646 & 7,241 & 0,013 & 0,084 & 0,465 \\
\hline Trauma & Nein & $59,7(37)$ & 9,321 & 8,532 & & & & 3,476 & 4,298 & & & \\
\hline Anfall und Epile & psie & & & & & & & & & & & \\
\hline Anzahl AED & 1 & $82,3(51)$ & 8,428 & 12,286 & 0,042 & 0,063 & 0,465 & 3,263 & 4,840 & 0,057 & 0,006 & 0,947 \\
\hline & $\geq 1$ & $17,7(11)$ & 9,637 & 10,976 & & & & 4,096 & 5,836 & & & \\
\hline Anfalls- & $\geq 1 / y$ & $66,1(41)$ & 9,056 & 4,103 & 0,046 & $-0,010$ & 0,974 & 4,103 & 5,650 & 0,046 & $-0,10$ & 0,940 \\
\hline trequenz & $<1 / y$ & $33,9(21)$ & 10,139 & 13,268 & & & & 3,644 & 5,760 & & & \\
\hline Generalisier- & Ja & $80,6(50)$ & 9,751 & 11,640 & 0,061 & $-0,027$ & 0,807 & 4,180 & 5,952 & 0,085 & $-0,051$ & 0,569 \\
\hline ter Antall & Nein & $19,4(12)$ & 8,055 & 8,944 & & & & 2,980 & 4,196 & & & \\
\hline
\end{tabular}

Kosten jedoch mit zunehmendem Abstand zur primären Erhebung. Aufgrund des für die Berechnungen verwendeten auf Tagespauschalen und Operationsminuten basierenden Ansatzes konnten, verglichen mit einem prospektiven $\mathrm{Mi}$ cro-costing-Ansatz, keine gesonderten räumlichen, apparativen, materiellen oder personellen Ausgaben berücksichtigt werden. Ebenso wurden keine generellen Anschaffungs- bzw. Vorhaltekosten berücksichtigt, da diese in Bezug auf krankheitsspezifische Versorgungs- kosten nicht von Interesse sind, sich in der DRG-Vergütung aber ebenfalls widerspiegeln sollten. Aufgrund der einheitlichen Vergütung stationärer Leistungen in Deutschland nach G-DRG sowie der standardisierten SOP- und leitlinienorientierten Behandlung von Frakturen erscheint ein Vergleich der Ergebnisse mit anderen Kliniken auf nationaler Ebene jedoch vertretbar.

Zusammenfassend sind die stationären, direkten Versorgungskosten von Patienten mit Verletzungen im Rahmen epileptischer Anfälle hoch und aufgrund ihrer Häufigkeit gesundheitsökonomisch relevant. Die auf Fallpauschalen basierende Vergütung der Kosten scheint überwiegend kostendeckend $\mathrm{zu}$ sein, analog zu bereits veröffentlichten Untersuchungen scheinen insbesondere bei Patienten mit langer Liegedauer oder intensivmedizinischer Versorgung Vergütungsprobleme $\mathrm{zu}$ bestehen. Weitere gezielte gesundheitsökonomische Analysen, z. B. mittels exakter Erfassung der einzelnen Kosten („micro-costing“) 
werden benötigt, um diesen Hinweisen weiter nachzugehen und eine verbesserte Kostendeckung medizinischer Maßnahmen durch das aktuelle, auf Fallpauschalen basierte Vergütungssystem nach G-DRG zu erreichen.

\section{Korrespondenzadresse}

\section{Dr. med. René D. Verboket, MHBA}

Klinik für Unfall-, Hand- und Wiederherstellungschirurgie, Goethe-Universität Frankfurt

Theodor-Stern-Kai 7, 60590 Frankfurt am Main, Deutschland

rene.verboket@kgu.de

Funding. Open Access funding provided by Projekt DEAL.

\section{Einhaltung ethischer Richtlinien}

Interessenkonflikt. R. D. Verboket, N. Mühlenfeld, J. Sterz, P. Störmann, Y. Balcik und L. M. Willems geben an, dass keine Interessenkonflikte bestehen. A. Strzelczyk erhielt Beratungs-, Referentenhonorare und/oder Unterstützung für Forschungsvorhaben von Arvelle Therapeutics, Desitin Arzneimittel, Eisai, GW Pharma, LivaNova, Marinus pharmaceuticals, Medtronic, UCB Pharma und Zogenix. I. Marzi gibt Förderung der AO-Foundation und der Deutschen Forschungsgemeinschaft an. F. Rosenow erhielt Beratungs- und Referentenhonorare von Arvelle Therapeutics, Desitin Arzneimittel, Eisai, GW Pharma, Medtronic, Novartis, UCB Pharma und Unterstützung für Forschungsvorhaben der Europäischen Union, des Landes Hessen im Rahmen des LOEWE-Programmes, des BMBF im Rahmen des ERAPerMed Programmes, des DetlevWrobel-Fonds für Epilepsieforschung und der DFG.

Für diesen Beitrag wurden von den Autoren keine Studien an Menschen oder Tieren durchgeführt. Für die aufgeführten Studien gelten die jeweils dort angegebenen ethischen Richtlinien.

Open Access. Dieser Artikel wird unter der Creative Commons Namensnennung 4.0 International Lizenz veröffentlicht, welche die Nutzung, Vervielfältigung, Bearbeitung, Verbreitung und Wiedergabe in jeglichem Medium und Format erlaubt, sofern Sie den/die ursprünglichen Autor(en) und die Quelle ordnungsgemäß nennen, einen Link zur Creative Commons Lizenz beifügen und angeben, ob Änderungen vorgenommen wurden.

Die in diesem Artikel enthaltenen Bilder und sonstiges Drittmaterial unterliegen ebenfalls der genannten Creative Commons Lizenz, sofern sich aus der Abbildungslegende nichts anderes ergibt. Sofern das betreffende Material nicht unter der genannten Creative Commons Lizenz steht und die betreffende Handlung nicht nach gesetzlichen Vorschriften erlaubt ist, ist für die oben aufgeführten Weiterverwendungen des $\mathrm{Ma}$ terials die Einwilligung des jeweiligen Rechteinhabers einzuholen.
Weitere Details zur Lizenz entnehmen Sie bitte der Lizenzinformation auf http://creativecommons.org/ licenses/by/4.0/deed.de.

\section{Literatur}

1. Beerhorst K, Tan IY, De Krom M et al (2013) Antiepileptic drugs and high prevalence of low bone mineral density in a group of inpatients with chronic epilepsy. Acta Neurol Scand 128:273-280

2. Benjamini Y, Hochberg Y (1995) Controlling the false discovery rate-a practical and powerful approach to multiple testing. J R Stat Soc Series B Stat Methodol 57:289-300

3. Bock JO, Brettschneider C, Seidl H et al (2015) Calculation of standardised unit costs from a societal perspective for health economic evaluation. Gesundheitswesen 77:53-61

4. Camfield C, Camfield P(2015) Injuries from seizures are a serious, persistent problem in childhood onset epilepsy: a population-based study. Seizure 27:80-83

5. Cevallos M, Egger M (2014) STROBE (STrengthening the reporting of observational studies in epidemiology). John Wiley \& Sons, Oxford, S169-179

6. Destatis (2020) Kapitel 4 - Gesundheit. In: Statistisches Jahrbuch 2019. Statistisches Bundesamt (Destatis), Wiesbaden

7. Fedorenko M, Wagner ML, Wu BY (2015) Survey of risk factors for osteoporosis and osteoprotective behaviors among patients with epilepsy. Epilepsy Behav 45:217-222

8. Fisher RS, Acevedo C, Arzimanoglou A et al (2014) ILAE official report: a practical clinical definition of epilepsy. Epilepsia 55:475-482

9. Frey K, Zöllner JP, Knake S, Oganian Y, Kay L, Mahr K, Keil F, Willems LW, Menzler K, Bauer S, SchubertBast S, Rosenow F, Strzelczyk A (2020) Risk incidence of fractures and injuries: a multicenter video-EEG study of 626 generalized convulsive seizures. JNeurol https://doi.org/10.1007/s00415020-10065-5

10. Friedman DE, Gilliam FG (2010) Seizure-related injuries are underreported in pharmacoresistant localization-related epilepsy. Epilepsia 51:43-47

11. Hamer HM, Spottke A, Aletsee C et al (2006) Direct and indirect costs of refractory epilepsy in a tertiary epilepsy center in Germany. Epilepsia 47:2165-2172

12. Hamer HM, Dodel R, Strzelczyk A et al (2012) Prevalence, utilization, and costs of antiepileptic drugs for epilepsy in Germany-a nationwide population-based study in children and adults. JNeurol 259:2376-2384

13. Harron K, Benchimol E, Langan S (2018) Using the RECORD guidelines to improve transparent reporting of studies based on routinely collected data. Int J Popul Data Sc 3:2

14. Knoll M, Otte I, Salloch S et al (2018) "something humane has been lost". Re-evaluation of the attitudes of senior physicians towards the G-DRG system - a qualitative normative analysis. Z Evid Fortbild Qual Gesundhwes 131-132:60-65

15. Krauth C, Hessel F, Hansmeier T et al (2005) Empirical standard costs for health economic evaluation in Germany-a proposal by the working group methods in health economic evaluation. Gesundheitswesen 67:736-746

16. Mahr K, Bergmann MP, Kay L, Möller L, Reif PS, Willems LM, Menzler K, Schubert-Bast S, Klein KM, Knake S, Rosenow F, Zöllner JP, Strzelczyk A (2020)
Prone, lateral, or supine positioning at seizure onset determines the postictal body position: A multicenter video-EEG monitoring cohort study. Seizure 76:173-178

17. Mühlenfeld N, Verboket RD, Söhling N, Pieper M, Paule E, ReifPS, Willems LM (2019) Fractures in Parkinson's Disease: injury patterns, hospitalization, and therapeutic aspects. Eur J Trauma Emerg Surg. https://doi.org/10.1007/s00068-019-01240-z

18. Newson R (2008) Identity of Somers' D and the rank biserial correlation coefficient

19. Olivier M, Boeker M, Hanser S et al (2019) The PKMS (German score for complex nursing measures) as paradigm shift in the G-DRG system: a retrospective observational study based on billing data. Gesundh ökon Qual manag 24:191-196

20. Riechmann J, Strzelczyk A, Reese JP et al (2015) Costs of epilepsy and cost-driving factors in children, adolescents, and their caregivers in Germany. Epilepsia 56:1388-1397

21. Riechmann J, Willems LM, Boor R et al (2019) Quality of life and correlating factors in children, adolescents with epilepsy, and their caregivers: a cross-sectional multicenter study from Germany. Seizure 69:92-98

22. Riessen R, Hermes C, Bodmann KF et al (2018) Reimbursement of intensive care services in the German DRG system : current problems and possible solutions. Med Klin Intensivmed Notfmed 113:13-23

23. Roeder N, Franz D, Siebert $H$ et al (2003) DRG-Anpassungsvorschläge der Fachgebiete Orthopädie und Unfallchirurgie für 2004. Z Orthop Unfall 141:379-385

24. Roeder N, Fiori W, Bunzemeier H (2020) Weiterentwicklungsperspektiven des G-DRG-Systems. In: Klauber J, Geraedts M, Friedrich J, Wasem J, Beivers A (Hrsg) Krankenhaus-Report 2020. Springer, Berlin, Heidelberg

25. Rosenow F, Bast T, Czech T et al (2016) Revised version of quality guidelines for presurgical epilepsy evaluation and surgical epilepsy therapy issued by the Austrian, German, and Swiss working group on presurgical epilepsy diagnosis and operative epilepsy treatment. Epilepsia 57:1215-1220

26. Schäfer A, Schöttker-Königer T (2015) Statistik und quantitative Methoden für Gesundheitsfachberufe

27. Schindler CR, Lustenberger T, Marzi I, Verboket RD (2020) Cost factor "outpatient wound care" in the emergency department: Cost-revenue deficit of outpatient wound treatment in a German university hospital. Unfallchirurg. https://doi.org/ 10.1007/s00113-020-00819-1

28. Schöffski O, Graf von der Schulenburg J-M (2007) Gesundheitsökonomische Evaluationen, 3. Aufl. Springer, Berlin

29. Simon M (2019) Das deutsche DRG-System: Weder Erfolgsgeschichte noch leistungsgerecht. In: Dietereich A, Braun B, Gerlinger T, Simon M (Hrsg) Geld im Krankenhaus. Springer VS, Wiesbaden

30. Somers RH (1962) A new asymmetric measure of association for ordinal variables. Am Sociol Rev 27:799-811

31. Strzelczyk A, Reese JP, Dodel R et al (2008) Cost of epilepsy: a systematic review. PharmacoEconomics 26:463-476

32. Strzelczyk A, Haag A, Reese JP et al (2013) Trends in resource utilization and prescription of anticonvulsants for patients with active epilepsy in Germany. Epilepsy Behav 27:433-438 


\section{Originalien}

33. Strzelczyk A, Hermsen A, Belke $M$ et al (2014) Incidence, hospitalization costs and risk factors of epilepsy-related injuries and accidents. Epilepsia 55:200-200

34. Strzelczyk A, Griebel C, Lux W et al (2017) The burden of severely drug-refractory epilepsy: a comparative longitudinal evaluation of mortality, morbidity, resource use and cost using German health insurance data. Front Neurol 8:712

35. Taylor S, Curri T, Lawless M et al (2014) Predicting resource utilization in burn treatment. J Burn Care Res 35(2):S235-246

36. Verboket R, Verboket C, Schoffski O et al (2019) Costs and proceeds from patients admitted via the emergency room with mild craniocerebral trauma. Unfallchirurg 122:618-625

37. Verboket RD, Sohling N, Marzi I et al (2019) Prevalence, risk factors and therapeutic aspects of injuries and accidents in women with epilepsy. Eur JTrauma Emerg Surg 45:375-381

38. Verboket RD, Muhlenfeld N, Woschek Metal (2020) Inpatient treatment costs, cost-driving factors and potential reimbursement problems due to fall-related fractures in patients with Parkinson's disease. Chirurg 91(5):421-427. https://doi.org/ 10.1007/s00104-019-01074-w

39. Vestergaard P (2005) Epilepsy, osteoporosis and fracture risk-a meta-analysis. Acta Neurol Scand 112:277-286

40. Vollmann J (2011) DRG-Vergütung und ärztliche Professionsethik. Wirtschaftsethik in der Medizin, S97-114

41. Waeschle RM, Hinz J, Bleeker F et al (2016) OR minute myth: guidelines for calculation of DRG revenues per OR minute. Anaesthesist 65:137-147
42. Willems LM, Richter S, Watermann N et al (2018a) Trends in resource utilization and prescription of anticonvulsants for patients with active epilepsy in Germany from 2003 to 2013-a ten-year overview. Epilepsy Behav 83:28-35

43. Willems LM, Watermann N, Richter $S$ et al (2018b) Incidence, risk factors and consequences of epilepsy-related injuries and accidents: a retrospective, single center study. Front Neurol 9:414

44. Willems LM, Hamer HM, Knake S et al (2019a) General trends in prices and prescription patterns of anticonvulsants in Germany between 2000 and 2017: analysis of national and cohort-based data. Appl Health Econ Health Policy 17:707-722

45. Willems LM, ReifPS, Knake Set al (2019b) Noncompliance of patients with driving restrictions due to uncontrolled epilepsy. Epilepsy Behav 91:86-89

Hier steht eine Anzeige. 